\title{
A New Explanation of Deflection Results of Charged Particles in High-velocity Motion in Magnetic Field·Correction of Lorentz Force
}

\author{
Kexin $\mathrm{Yao}^{1}$ \\ ${ }^{1}$ Institute of Mechanical Engineering of Shanxi Province, Xian Metering Institution, Xian, P. R. China \\ Correspondence: Kexin Yao, Institute of Mechanical Engineering of Shanxi Province, Xian Metering Institution, \\ Xian, P. R. China. Tel: 86-134-8462-6424. E-mail: yayydwpq@163.com,029bmsp@163.com
}

Received: June 15, 2014 Accepted: July 23, 2015 Online Published: August 12, 2015

doi:10.5539/apr.v7n5p13 URL: http://dx.doi.org/10.5539/apr.v7n5p13

\begin{abstract}
It is incorrect to only apply mass change or time change in explanation of the deflection result of charged particle in high velocity motion in magnetic field. A scientific and correct method is to change mass and time at the same time. However, it is impracticable to necessitate force formula in simultaneous change of mass and time. The paper makes correction of Lorentz Force formula based on analysis method for acting force in electric field, and launches into a new understanding of deflection result of charged particles in high velocity motion in magnetic field according to the corrected Lorentz Force formula.
\end{abstract}

Keywords: charged particle, magnetic field, deflection, Lorentz Force, mass change, time change, electric field force

\section{Introduction}

It is known to all in the field of physics that charged particle moving through magnetic field shall undergo deflection. The general explanation of the phenomenon is that when a charged particle with a charge of $\mathrm{Q}$ moves through magnetic field at the velocity of $\mathrm{V}$ along the direction of $\mathrm{x}$, Q shall undergo Lorentz Force $F=B Q V$ ( $B$ - magnetic induction) along the direction normal to V. F shall make the charged particle with a mass of $\mathrm{M}$ and a charge of $\mathrm{Q}$ generate an acceleration $\mathrm{a}=\mathrm{F} / \mathrm{M}$ along the direction normal to $\mathrm{V}$ (direction of $\mathrm{Y}$ ) to make $\mathrm{Q}$ generate migration $\mathrm{Y}$ along the direction normal to $\mathrm{V}$, namely deflection $\mathrm{Y}$. Suppose the time for $\mathrm{Q}$ to move through magnetic field is $\mathrm{t}$, then the expression for deflection $\mathrm{Y}$ is as follows:

$$
Y=\frac{1}{2} a t^{2}=\frac{F}{2 M} t^{2}
$$

Where (1) is derived under the condition that the direction of $\mathrm{F}$ being unchanged; although when particle moves through magnetic field, directions of velocity $\mathrm{V}$ and $\mathrm{F}$ ( $\mathrm{F}$ is normal to $\mathrm{V}$ ) are changed to a certain extent, since $\mathrm{t}$ is too short, the changes in $\mathrm{F}$ and $\mathrm{V}$ are also minor, accordingly, we can consider that there is no change in the directions of $\mathrm{F}$ and $\mathrm{V}$ and that the error of $\mathrm{Y}$ may be ignored.

Where (1) is practical on condition that velocity $\mathrm{V}$ be low, but if $\mathrm{V}$ is very high, there is obvious deviation between the calculated result of (1) and measured result, higher V shall bring about larger deviation, experiment shows that when $\mathrm{V}$ is very high, deflection distance shall be:

$$
Y=\frac{F t^{2}}{2 M} \sqrt{1-\frac{V^{2}}{C^{2}}} \quad(\mathrm{c}-\text { light velocity) }
$$

At present, there are several explanations for the experimental result indicated by expression (2), it is observed that all these explanations are unscientific, the reasons for which are as follows:

The most general explanation is that when charged particle is in high velocity motion, according to special relativity, the mass $\mathrm{M}$ of the charged particle shall increase to $M^{\prime}=M / \sqrt{1-V^{2} / C^{2}}$, replace $\mathrm{M}$ in expression (1) with $M^{\prime}$ to obtain the practical expression (2).

Another method is to carry out analysis based on change of particle momentum. Suppose the migration velocity of particle along direction of $\boldsymbol{Y}$ is $u$ and particle mass is $M^{\prime}=M / \sqrt{1-V^{2} / C^{2}}$ and particle momentum along direction of $\boldsymbol{Y}$ is $P=M^{\prime} u=M u / \sqrt{1-V^{2} / C^{2}}$ and the acting force on particle along direction of $\boldsymbol{Y}$ is 


$$
\begin{gathered}
F=\frac{d P}{d t}=\frac{M}{\sqrt{1-V^{2} / C^{2}}} \frac{d u}{d t} \\
\frac{d u}{d t}=\frac{F \sqrt{1-V^{2} / C^{2}}}{M} \\
Y=\int_{o}^{t} d t \int_{o}^{t} \frac{d u}{d t} \cdot d t=\frac{F t^{2}}{2 M} \sqrt{1-V^{2} / C^{2}}
\end{gathered}
$$

It is observed that $d u / d t$ in the analysis method is constant acceleration $a$ in substance, the deflection it determined is still $Y=a t^{2} / 2$, and that the key to the analysis method is $M^{\prime}=M / \sqrt{1-V^{2} / C^{2}}$, there is no substantial difference between this method and the first method. Compared with the first method, it is obvious that this method is lack of its physical significance.

There is another method specified in Berkeley Physics, the method defines that $\Delta \tau$ is particle clock time and that mass is rest mass M. $\Delta Y$ is displacement of particle in $\Delta t$ along direction of $\boldsymbol{Y}$, momentum: $P=M \Delta Y / \Delta \tau$. It is observed that the time for particle to undergo displacement along direction of $\boldsymbol{Y}$ is $\Delta \tau$, according to special relativity, there is $\Delta \tau=\Delta t \sqrt{1-V^{2} / C^{2}}$, therefore there is $P=M \Delta Y / \Delta \tau=M \Delta Y / \Delta t \sqrt{1-V^{2} / C^{2}}$. Since the displacement velocity of particle along direction of $\boldsymbol{Y}$ is $V=\Delta Y / \Delta t$, accordingly, $P=M V / \sqrt{1-V^{2} / C^{2}}$, the momentum given in the result is identical with that obtained in the previous method. It is natural to come to a conclusion identical with that obtained by expression (2) according to the deduction steps given in the previous method.

It is observed that the first and second methods only apply mass change, although they do not mention that they only adopt mass change, in fact, time is unchanged, being a constant; the third method only applies time change, specifying that mass is unrelated to motion, being a constant. However, according to special relativity, the mass of particle in high velocity motion and time are changed simultaneously, accordingly, it is improper to suppose mass is unchanged and time is unchanged, because it is in violation of special relativity and practical result.

In accordance with special relativity, the correct method in analyzing the deflection of grain is to use both mass conversion $M^{\prime}=M / \sqrt{1-V^{2} / C^{2}}$ and time conversion $t^{\prime}=t \sqrt{1-V^{2} / C^{2}}$. Take $M^{\prime}$ and $t^{\prime}$ into Formula (1), and then

$$
Y=\frac{F t^{\prime 2}}{2 M^{\prime}}=\frac{F t^{2}}{2 M}\left(1-\frac{V^{2}}{C^{2}}\right)^{3 / 2}
$$

It is obvious that Formula (3) is not in line with the result of (2). Why? It is certain that Formula (1) is the basic formula of mechanics, and this shall be affirmed; besides, it is without doubt that quality and time shall be conversed at the same time. Therefore, the only possibility is that Formula (3) does not coincide with reality, and it must be Lorentz Force, $\mathrm{F}$ that fails to show the actual power.

Lorentz Force $F=B Q V$ is only the experimental conclusion that charged particles of low runner are stressed in uniform magnetic field. It doesn't have necessary theoretical explanation. To analyze Lorentz Force is in line with reality or not, it is necessary to know the reason and change rules of Lorentz Force. Therefore, we have to illustrate the root of Lorentz Force from the theory and then it can analyze and solve the actual problems in Formula (3).

\section{The Original Analysis of Lorentz Force}

It is shown in experiments that electric charge will be influenced by force in electric field, and the electric field can be considered as the only origin of charge force. Therefore, the electric field force can be considered as the basic point in analyzing Lorentz Force.

It can be known from the superposition principle of static electric field that the distance between two positron and negatron in space, the total electric field of positron and negatron must be equal to the vector sum when the positron and negatron exist alone. It can be deduced that any free electron in conductor (hereinafter called negatron) and the proton that is equal with free electron in electric quantity (hereinafter called positron) has their own electric field. Therefore, when there is current in conductor, namely the macroscopic motion of negatron in conductor exists, the electric field of negatron must move with negatron. And the macroeconomic effect is that the negative electric field moving around conductor exists along with the static positive electric field. In terms of magnet, the electron that spins in same direction in magnetic domain is similar to the negatron of macroscopic motion in 
conductor. Therefore, there are negative electric field of macroscopic motion and static positive electric field in magnetic domain.

First of all, we shall analyze the relationship between magnetic field and electric field. It is showed in Biot-Savart theorem that, the feeling strength of any current element $I d \boldsymbol{l}$ (small lines on the wire that in the same direction of current) at the place of $r$ is

$$
d \boldsymbol{B}=\frac{\mu_{0}}{4 \pi} \cdot \frac{I d \boldsymbol{l}}{r^{2}} \times \boldsymbol{r}_{0} \quad \text { (Unit vector in the direction of } \boldsymbol{r}_{0}-\boldsymbol{r} \text { ) }
$$

Since I is actually formed by free electron in the wire in the speed of V (in the opposite direction of current I). Taking $-\tau$ as the linear charge density in the wire, then $I d \boldsymbol{l}=-\tau(-\boldsymbol{V}) d l=d Q \boldsymbol{V}$ (the quantity of free electron in $d Q-d l)$ and because $\mu_{0} \varepsilon_{0}=1 / C^{2}, K=1 / 4 \pi \varepsilon_{0}$, then

$$
\begin{gathered}
\frac{\mu_{0}}{4 \pi}=\frac{K}{C^{2}} \\
d \boldsymbol{B}=\frac{K}{C^{2}} \cdot \frac{d Q \boldsymbol{V}}{r^{2}} \times \boldsymbol{r}_{0}=\frac{1}{C^{2}} \boldsymbol{V} \times K \frac{d Q}{r^{2}} \boldsymbol{r}_{0} \\
=\frac{1}{C^{2}} \boldsymbol{V} \times d \boldsymbol{E}=\mu_{0} \varepsilon_{0} \boldsymbol{V} \times d \boldsymbol{E} .
\end{gathered}
$$

In the upper formula, $d \boldsymbol{E}$ is the electric field intensity of $d Q$ at the place of R. and the formula can be written as

$$
\boldsymbol{B}=\frac{1}{C^{2}} \boldsymbol{V} \times \boldsymbol{E}=\mu_{0} \varepsilon_{0} \boldsymbol{V} \times \boldsymbol{E}
$$

(4) The formula shows that the feeling strength at some place is equal to the vector product of kinematic velocity $\mathrm{V}$ and the electric field intensity. The formula shows that magnetic field is a moving electric field.

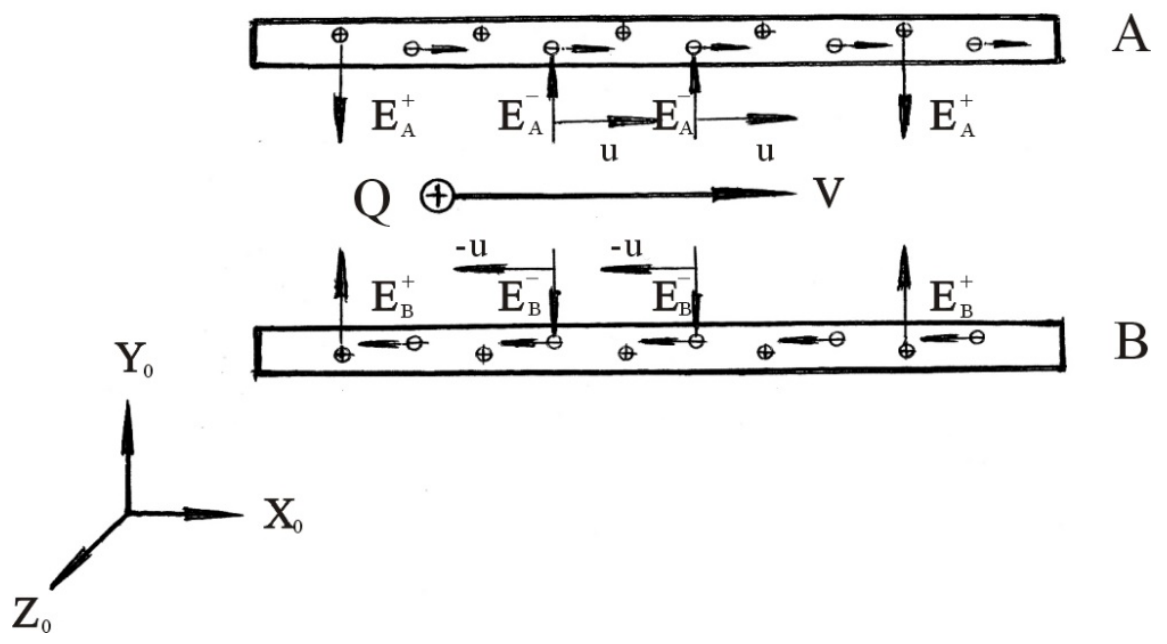

Figure 1. Origin analysis of Lorentz Force

It is defined that flat consisting of wires with current is the current surface, in Figure 1, A and B indicates two sections of "infinity" current surface. A and B current surfaces are made up of two common wire with current, and the current of $\mathrm{A}$ and $\mathrm{B}$ is equal but in opposite direction. In Figure, $\boldsymbol{E}_{\boldsymbol{A}}^{-}$and $\boldsymbol{E}_{\boldsymbol{B}}^{-}$shows the electric field intensity that negatron electron in motion in current surface; $\boldsymbol{E}_{\boldsymbol{A}}^{+}$and $\boldsymbol{E}_{\boldsymbol{B}}^{+}$indicate the electric field intensity that produced by static positive electron that is equal to negatron in electric quantity.

It shall count out the feeling strength between two current surfaces A and B. It can be found out from Formula (4) that, B is originated from moving electric field. The motion of $\boldsymbol{E}_{\boldsymbol{A}}^{+}$and $\boldsymbol{E}_{\boldsymbol{B}}^{+}$has nothing to do with B. And then we only analyze the electric field of $\boldsymbol{E}_{\boldsymbol{A}}^{-}$that moves in the speed of $\mathrm{V}$ and then electric field of $\boldsymbol{E}_{\boldsymbol{B}}^{-}$in the speed of $-\mathrm{V}$. Obviously, $\boldsymbol{E}_{\boldsymbol{A}}^{-}$and $\boldsymbol{E}_{\boldsymbol{B}}^{-}$is equal but in opposite direction, namely $\boldsymbol{E}_{\boldsymbol{A}}^{-}=\boldsymbol{E}_{\boldsymbol{B}}^{-}$. where $E_{\boldsymbol{0}}$ is the absolute value of $\boldsymbol{E}_{A}^{-}$and $\boldsymbol{E}_{B}^{-}$. 
It shall count out the feeling strength of $\boldsymbol{B}_{\boldsymbol{A}}$ from $\boldsymbol{E}_{\boldsymbol{A}}^{-}$, and from Formula (4)

$$
\boldsymbol{B}_{A}=\mu \varepsilon_{0} \boldsymbol{u} \times \boldsymbol{E}_{A}^{-}=\mu_{0} \varepsilon_{0} u E_{0} \boldsymbol{Z}_{0} \quad\left(Z_{0}\right. \text { is the unit vector in the direction of Z) }
$$

The feeling strength $\boldsymbol{B}_{B}$ produced by $\boldsymbol{E}_{\boldsymbol{B}}^{-}$is

$$
\boldsymbol{B}_{\boldsymbol{B}}=\mu_{0} \varepsilon_{0}-\boldsymbol{u} \times \boldsymbol{E}_{B}^{-}=\mu_{0} \varepsilon_{0}-\boldsymbol{u} \times\left(-\boldsymbol{E}_{A}^{-}\right)=\mu_{0} \varepsilon_{0} u E_{0} \boldsymbol{Z}_{0}
$$

The total feeling strength produced by current surface A and B is

$$
\boldsymbol{B}=\boldsymbol{B}_{A}+\boldsymbol{B}_{B}=2 \mu_{0} \varepsilon_{0} u E_{0} \boldsymbol{Z}_{0}
$$

Figure 1, $\mathrm{Q}$ is the charge particle with positive electricity in the speed of $\mathrm{V}$ paralleling two electricity surfaces. The Lorentz Force that is bore by grant with $\mathrm{Q}$ charge is

$$
\boldsymbol{F}=Q \boldsymbol{V} \times \boldsymbol{B}=2 \mu_{0} \varepsilon_{0} V u E_{0} Q\left(-\boldsymbol{Y}_{0}\right) \quad\left(\boldsymbol{Y}_{0} \text { is the unit vector in the direction of } \mathrm{Y}\right)
$$

When we make analysis of the source of Lorentz Force, it will be our natural selection for us to start with the source of force $\mathrm{Q}$. There is no doubt that the only source of $\mathrm{Q}$ force is under the effect from electric field. There are not only electric field $\boldsymbol{E}_{\boldsymbol{A}}^{-}$and $\boldsymbol{E}_{\boldsymbol{B}}^{-}$but $\boldsymbol{E}_{\boldsymbol{A}}^{-} 、 \boldsymbol{E}_{\boldsymbol{B}}^{+}$as well between the two current surface A and B; because the current of $\boldsymbol{E}_{A}^{+}$and $\boldsymbol{E}_{B}^{+}$is the same intensive with contrary direction and they has the same relative velocity to any moving Q, $\boldsymbol{E}_{\boldsymbol{A}}^{+}$and $\boldsymbol{E}_{\boldsymbol{B}}^{+}$will have a constant force on force Q with contrary directions and their forces will be cancelled out, namely the effect of $\boldsymbol{E}_{\boldsymbol{A}}^{+}$and $\boldsymbol{E}_{\boldsymbol{B}}^{+}$can not be considered on force Q. Without consideration of $\boldsymbol{E}_{\boldsymbol{A}}^{+}$and $\boldsymbol{E}_{\boldsymbol{B}}^{+}$, the force of Q is only sourced from $\boldsymbol{E}_{\boldsymbol{A}}^{-}$and $\boldsymbol{E}_{\boldsymbol{B}}^{-}$possibly.

Let us study the force on Q from $\boldsymbol{E}_{\boldsymbol{A}}^{-}$firstly, it is can be known that the relative velocity of $\boldsymbol{E}_{\boldsymbol{A}}^{-}$and Q is $V_{A}=V-u_{0}$ from Figure 1; According to Special Relativity, the electric field $\boldsymbol{E}_{\boldsymbol{A}}^{-}$with $V_{A}$ moving on will shrink in the proportion of $1 / \sqrt{1-V_{A}^{2} / C^{2}}$ in the direction of $V_{A}$ and the field density is inversely proportional to the distance of power line, therefore the filed density shall be magnified as:

$$
\boldsymbol{E}_{A}^{\prime}=\boldsymbol{E}_{A}^{-} / \sqrt{1-V_{A}^{2} / C^{2}}=\boldsymbol{E}_{A}^{-} / \sqrt{1-(V-u)^{2} / C^{2}}
$$

According to binomial series, $1 / \sqrt{1-X^{2}}$ can be expanded into $1+X^{2} / 2+3 X^{4} / 8+5 X^{6} / 16+\ldots \ldots$, when $\mathrm{x}$ is relative small, it can be neglected including $X^{4}$, and $X^{6} \ldots$..etc., it is $1 / \sqrt{1-X^{2}}=1+X^{2} / 2$. Under general condition, $\mathrm{V}$ and $\mathrm{u}$ is far less than $\mathrm{C}$, therefore $\boldsymbol{E}_{\boldsymbol{A}}^{-}$in the above formula can be converted into:

$$
\boldsymbol{E}_{\boldsymbol{A}}^{-}=\boldsymbol{E}_{\boldsymbol{A}}^{-}\left[1+(V-u)^{2} / 2 C^{2}\right]=E_{0}\left[1+(V-u)^{2} / 2 C^{2}\right] \boldsymbol{Y}_{0}
$$

Let us turn to $\boldsymbol{E}_{B}^{-}$, it is can be known that the relative velocity of $\boldsymbol{E}_{B}^{-}$and Q is $V_{B}=V+u$ and it can be got from the explanations mentioned above as:

$$
\boldsymbol{E}_{B}^{-}{ }^{\prime}=\boldsymbol{E}_{\boldsymbol{B}}^{-} / \sqrt{1-(V+u)^{2} / C^{2}}=E_{0}\left[1+(V+u)^{2} / 2 C^{2}\right]\left(-\boldsymbol{Y}_{0}\right)
$$

For the moving $\mathrm{Q}$, the total field intensity between the current surface A and B is

$$
\begin{aligned}
\boldsymbol{E}^{\prime} & =\boldsymbol{E}_{\boldsymbol{A}}^{\prime}+\boldsymbol{E}_{B}^{\prime} \\
& =E_{0}\left[1+\frac{(V-u)^{2}}{2 C^{2}}\right] \boldsymbol{Y}_{0}+E_{0}\left[1+\frac{(V+u)^{2}}{2 C^{2}}\right]\left(-\boldsymbol{Y}_{0}\right) \\
& =E_{0}\left[1+\frac{(V+u)^{2}}{2 C^{2}}-1-\frac{(V-u)^{2}}{2 C^{2}}\right]\left(-\boldsymbol{Y}_{0}\right) \\
& =\frac{2}{C^{2}} V u E_{0}\left(-\boldsymbol{Y}_{0}\right)=2 \mu_{0} \varepsilon_{0} V u E_{0}\left(-\boldsymbol{Y}_{0}\right)
\end{aligned}
$$

The force on $\mathrm{Q}$ is:

$$
\boldsymbol{F}=\boldsymbol{E}^{\prime} Q=2 \mu_{0} \varepsilon_{0} V u E_{0} Q\left(-\boldsymbol{Y}_{0}\right)
$$


It is obvious that the formula is just the Lorentz Force Formula (5) mentioned before. It can be concluded from the above analysis that: when Q is moving in magnetic field at the velocity $\mathrm{V}$, one moving electric field with two current surface $\boldsymbol{E}_{\boldsymbol{A}}^{-}$and $\boldsymbol{E}_{\boldsymbol{B}}^{-}$will be formed; for Q, their velocity are $V+u$ and $V$-u respectively and the electric field of $V+u$ will shrink greater with more intensive field; when the electric filed of $V$ - $u$ shrinks with less intensive filed intensity, one synthesis electric field will be formed by their differentiated filed intensity, whose force on Q will be just Lorentz Force.

\section{Correction of Lorentz Force}

It can be known from the analysis on the derivation Formula of above-mentioned Lorentz Force that the derivation process will not hold when the velocity of $\mathrm{Q}$ is very large (almost reaching the velocity of light $\mathrm{C}$ ); there are two reasons, one is that when $\mathrm{V}$ is very large, $V^{4} / C^{4}$ and $V^{6} / C^{6} \ldots \ldots$ can not be neglected, and the above derivation will not hold naturally; the other is that when $\mathrm{V}$ is very large, its velocities can not be added or subtracted directly, namely $V_{A} \neq V-u$ and $V_{B} \neq V+u$; therefore, the above derivation will not hold.

Now, let us make further analysis on the force of charged particle in high velocity motion (expressed with electric charge Q) in accordance with the synthesis velocity method given in Special Relativity.

The synthesis velocity mentioned in Special Relativity refers to that in the direction of X, Y and Z. For the condition given in Figure 1, the velocity on direction $\mathrm{Z}$ does not exist and migration velocity on direction $\mathrm{Y}$ is very slow, that's to say they can be neglected owing to the comparison with V. Therefore, it can be considered that there is also no velocity component on direction $\mathrm{Y}$ and we only need to make analysis of the synthesis velocity on direction $\mathrm{X}$ whose formula is given as:

$$
V_{x}=\frac{u_{x}+v}{1+u_{x} v / C^{2}}
$$

In the formula, $v$ is the moving velocity of the other inertial system $Z^{\prime}$ observed from inertial system $Z$ with the motionless of relative subject $\mathrm{M} ; u_{x}$ is the moving velocity relative to subject $\mathrm{N}, V_{x}$ is the relative velocity speed of $M$ and N. For Figure 1, we would like to conclude the force on Q (subject M), namely Q is of inertial system of $Z$ and two current surface is of the inertial system of $Z^{\prime}$, The velocity of $Z^{\prime}$ (two current surface) observed from $Z^{\prime}$ is $-V$, namely $v=-V$, and the motion velocity of $E_{A}^{-}$(subject N1) observed from $Z^{\prime}$ (two current surface) is $\mathrm{u}$, namely $u_{x}=u$; The velocity speed of Q (subject M) relative to $E_{A}^{-}$(subject N1) is in accordance with Formula (6)

$$
\boldsymbol{V}_{A}=\frac{u-v}{1-u v / C^{2}}
$$

However, the motion velocity of $E_{B}^{-}$observed from $Z^{\prime}$ (two current surface) is $-u$, namely $u_{x}=-u$; The velocity speed of Q (subject M) relative to $E_{B}^{-}$(subject N1) is in accordance with Formula (6)

$$
V_{B}=\frac{-u-v}{1+u v / C^{2}}
$$

$\boldsymbol{E}_{A}^{-}$moving at the speed of $\boldsymbol{V}_{\boldsymbol{A}}$ will shrink for Q, it shall be magnified as:

$$
\boldsymbol{E}_{A}^{-'}=\boldsymbol{E}_{A}^{-} / \sqrt{1-V_{A}^{2} / C^{2}}
$$

Put $\boldsymbol{V}_{\boldsymbol{A}}$ concluded from (7) in the following formula:

$$
\begin{gathered}
\boldsymbol{E}_{A}^{-}=\frac{\boldsymbol{E}_{A}^{-}}{\sqrt{1-\left(\frac{u-V}{1-u V / C^{2}}\right)^{2} / C^{2}}}=\frac{\boldsymbol{E}_{A}^{-}}{\sqrt{1-\frac{C^{4}(u-V)^{2}}{\left(C^{2}-u V\right)^{2}} / C^{2}}} \\
=\frac{\left(C^{2}-u V\right) \boldsymbol{E}_{A}^{-}}{\sqrt{\left(C^{2}-u V\right)^{2}-C^{2}(u-V)^{2}}}=\frac{\left(C^{2}-u V\right) \boldsymbol{E}_{A}^{-}}{\sqrt{C^{4}+u^{2} V^{2}-C^{2} u^{2}-C^{2} V^{2}}} \\
=\frac{\left(C^{2}-u V\right) \boldsymbol{E}_{A}^{-}}{\sqrt{\left(C^{2}-V^{2}\right)\left(C^{2}-u^{2}\right)}}=\frac{\left(C^{2}-u V\right) \boldsymbol{E}_{A}^{-}}{C^{2} \sqrt{\left(1-V^{2} / C^{2}\right)\left(1-u^{2} / C^{2}\right)}}
\end{gathered}
$$


The movement velocity of negative electron is far smaller than $\mathrm{C}$ ( electron in magnetic domain is also smaller) in the moving electric field formed in two current surfaces A and B; therefore $u^{2} / C^{2}$ is very small, which can be reckoned as $1-u^{2} / C^{2} \approx 1$, and then the above formula can be converted into:

$$
\boldsymbol{E}_{A}^{\prime}=\frac{\left(C^{2}-u V\right) \boldsymbol{E}_{A}^{-}}{C^{2} \sqrt{1-V^{2} / C^{2}}}
$$

For $\boldsymbol{E}_{B}^{-}, \boldsymbol{E}_{B}^{-}$moving at the speed of $V_{B}$ shall be shrank and magnified as:

$$
\boldsymbol{E}_{B}^{\prime}=\boldsymbol{E}_{B}^{-} / \sqrt{1-V_{B}^{2} / C^{2}}
$$

Put the $V_{B}$ got in Formula (8) into the following formula:

$$
\begin{aligned}
\boldsymbol{E}_{\boldsymbol{B}}^{-} & =\frac{\boldsymbol{E}_{\boldsymbol{B}}^{-}}{\sqrt{1-\left(\frac{u+V}{1+u V / C^{2}}\right)^{2} / C^{2}}}=\frac{\boldsymbol{E}_{\boldsymbol{B}}^{-}}{\sqrt{1-\frac{C^{4}(u+V)^{2}}{\left(C^{2}+u V\right)^{2}} / C^{2}}} \\
& =\frac{\left(C^{2}+u V\right) \boldsymbol{E}_{\boldsymbol{B}}^{-}}{\sqrt{\left(C^{2}+u V\right)^{2}-C^{2}(u+V)^{2}}}=\frac{\left(C^{2}+u V\right) \boldsymbol{E}_{\boldsymbol{B}}^{-}}{\sqrt{C^{4}+u^{2} V^{2}-C^{2} u^{2}-C^{2} V^{2}}} \\
& =\frac{\left(C^{2}+u V\right) \boldsymbol{E}_{\boldsymbol{B}}^{-}}{\sqrt{\left(C^{2}-V\right)\left(C^{2}-u^{2}\right)}}=\frac{\left(C^{2}+u V\right) \boldsymbol{E}_{\boldsymbol{B}}^{-}}{C^{2} \sqrt{1-V^{2} / C^{2}}}
\end{aligned}
$$

The field intensity felt by $\mathrm{Q}$ is:

$$
\begin{aligned}
\boldsymbol{E}^{\prime} & =\boldsymbol{E}_{B}{ }^{\prime}+\boldsymbol{E}_{A}^{\prime} \\
& =\frac{\left(C^{2}+u V\right) \boldsymbol{E}_{B}^{-}}{C^{2} \sqrt{1-V^{2} / C^{2}}}+\frac{\left(C^{2}-u V\right) \boldsymbol{E}_{A}^{-}}{C^{2} \sqrt{1-V^{2} / C^{2}}}
\end{aligned}
$$

Owing to $\boldsymbol{E}_{\boldsymbol{A}}^{-}=-\boldsymbol{E}_{\boldsymbol{B}}^{-}$, the above formula can be converted into:

$$
\begin{aligned}
\boldsymbol{E}^{\prime} & =\frac{\left(C^{2}+u V\right) \boldsymbol{E}_{B}^{-}}{C^{2} \sqrt{1-V^{2} / C^{2}}}-\frac{\left(C^{2}-u V\right) \boldsymbol{E}_{B}^{-}}{C^{2} \sqrt{1-V^{2} / C^{2}}} \\
& =\frac{2 u V \boldsymbol{E}_{B}^{-}}{C^{2} \sqrt{1-V^{2} / C^{2}}}=\frac{2 \mu_{0} \varepsilon_{0} u V E_{0}}{\sqrt{1-V^{2} / C^{2}}} \cdot\left(-\boldsymbol{Y}_{0}\right)
\end{aligned}
$$

For the sake of telling the force F, namely Lorentz Force F, exerted by electric field when Q is moving at medium and low speed, the force $\boldsymbol{F}_{V}$ on Q exerted by electric field can be concluded from Formula (11):

$$
\boldsymbol{F}_{V}=\boldsymbol{E}^{\prime} Q=\frac{2 \mu_{0} \varepsilon_{0} V u E_{0} Q}{\sqrt{1-V^{2} / C^{2}}}\left(-\boldsymbol{Y}_{0}\right)
$$

It is shown from former Formula (5) that Lorentz Force on Q is $\boldsymbol{F}=2 \mu_{0} \varepsilon_{0} V u E_{0} Q\left(-\boldsymbol{Y}_{0}\right)$ when it is moving at medium and low speed, and put it into Formula (12):

$$
\boldsymbol{F}_{V}=\frac{\boldsymbol{F}}{\sqrt{1-V^{2} / C^{2}}}=\frac{Q \boldsymbol{V} \times \boldsymbol{B}}{\sqrt{1-V^{2} / C^{2}}}
$$

The Formula (13) can be used for the correction of the formula of Lorentz Force. It is obvious that Formula (13) is also qualified for that at both high and low speed when Q is moving in magnetic field at medium and low speed. 


\section{Interpretation of Charged Particles' Deflection with the help of Corrected Formula for Lorentz Force}

The corrected Lorentz Force, $\boldsymbol{F}_{V}$, on Q got from above analysis is just the electric field force, which is relatively static to Q; it will move together with Q at the same speed V. For the observer with static relative magnetic field (two current surfaces), force $\boldsymbol{F}_{V}$ is a moving one. I have mentioned in "Set up Invariable Axiom of Force Equilibrium and Solve Problems about Transformation of Force and Gravitational Mass" (References. 3) that the force on moving subject will also change like its length, mass and time. The conversion formula for the force moving at the speed $\mathrm{V}$ is:

$$
\boldsymbol{F}^{\prime}=\boldsymbol{F} \sqrt{\frac{1-\cos ^{2} \theta V^{2} / C^{2}}{1-V^{2} / C^{2}}}
$$

In the formula, $\theta$ is the included angle between $\mathrm{F}$ and $\mathrm{V}$.

For $\boldsymbol{F}_{V}$ analyzed in Figure 1, the included angle between $\boldsymbol{F}_{V}$ and $\mathrm{V}$ is $\theta=90^{\circ}$ and $\cos \theta=0$, therefore the force $\boldsymbol{F}_{V}$ can be converted into in accordance with the Formula (14):

$$
\boldsymbol{F}_{V}^{\prime}=\boldsymbol{F}_{V} / \sqrt{1-V^{2} / C^{2}}
$$

Put Formula (13) into this Formula (13)

$$
\boldsymbol{F}_{V}^{\prime}=\frac{\boldsymbol{F}}{1-V^{2} / C^{2}}
$$

$\boldsymbol{F}_{V}{ }^{\prime}$ is just the real force exerted on charged particle we would like to discuss, put real $\boldsymbol{F}_{V}{ }^{\prime}$ into Formula (3) instead of F:

$$
Y=\frac{F_{V}^{\prime} t^{2}}{2 M}\left(1-\frac{V^{2}}{C^{2}}\right)^{3 / 2}=\frac{F t^{2}}{2 M} \sqrt{1-V^{2} / C^{2}}
$$

It is obvious that the deflection distance $\mathrm{Y}$ is just as determined in experiment room, namely true deflection distance Formula (2).

It is shown from the above analysis that the problem in connection with the deflection of charged particle moving at high speed can be solved reasonably provided that its mass and time are changed at the same time with the help of corrected Lorentz Force in combination with force transformation.

\section{Making Analysis of Particles' Deflection from the Inertial System of Relative Static of Charged Particles}

According to the relativity principle given in Special Relativity, for the observers of inertial system Z (inertial system $Z^{\prime}$ moving at the same speed of Q) and inertial system $Z^{\prime}$ (relative magnetic field or static surface), it is of no doubt that they will have the same result of the deflection on Q passing through the magnetic field on direction $\mathrm{Y}$.

The, former analysis is the conclusion of the observer relatively static to $Z^{\prime}$, for this conclusion, the real force $\boldsymbol{F}_{V}$ is got with the help of corrected Lorentz Force and changed combined power, moreover the analysis result compliance with fact is concluded. $\mathrm{Z}$ is moving relatively to $Z^{\prime}$ at the speed $\mathrm{V}$, it can be reckoned that $\boldsymbol{F}_{V}$ is static relatively to $\mathrm{Z}$ (with slight movement of $\boldsymbol{F}_{V}$ on direction Y neglected) there is no need for changing $\boldsymbol{F}_{V}$ any longe; Whether the change of this kind of conditions will have any influence on our analysis of the corresponding deflection? Let us make detailed analysis for it.

For observer $Z, Q$ is static and one magnetic field (inertial system $Z^{\prime}$ ) is passing through $\mathrm{Q}$ at the speed $-V$, one downward force $\boldsymbol{F}_{V}$ is be exerted on $\mathrm{Q}$, and moreover distance $\mathrm{Y}$ is moved downwards (deflection); because the speed of $\mathrm{Q}$ on direction $\mathrm{Y}$ is very slow, the mass of $\mathrm{Q}$ is still static one, namely no change is occurred to it. Besides the force on Q is also a static one, namely $\boldsymbol{F}_{V}=F / \sqrt{1-V^{2} / C^{2}}$ (corrected Lorentz Force), of course $\boldsymbol{F}_{V}$ will not change. The accelerated speed of $\mathrm{Q}$ on direct $\mathrm{Y}$ is $a=F_{V} / m$. Provided the time $\mathrm{Q}$ passing through the magnetic field is $t_{V}$ observed by $\mathrm{Z}$, the deflection distance of $\mathrm{Q}$ on direction $\mathrm{Y}$ is:

$$
Y=\frac{1}{2} a t_{V}^{2}=\frac{F t_{V}^{2}}{2 m \sqrt{1-V^{2} / C^{2}}}
$$

What is the relationship between $t_{V}$ and $Z^{\prime}$ observed by Z? First of all, we shall make sure that the absolute moving speed $\mathrm{V}$ and $-V$ of $\mathrm{Z}$ and $Z^{\prime}$ is the same and $\mathrm{Z}$ observes $\mathrm{t}$ is the time $\mathrm{Q}$ passing the magnetic field at the speed $\mathrm{V}$, namely $t=L / V$; for $\mathrm{Z}$, the magnetic in $\mathrm{L}$ wide is moving at the speed of $-V$, according to the Relativity Principle given in Special Theory, the width of the magnetic field moving at the speed $-V$ is 
$L^{\prime}=L \sqrt{1-V^{2} / C^{2}}$; therefore the time used by the magnetic passing through $\mathrm{Q}$ from the observation of $\mathrm{Z}$ is $t_{V}=L^{\prime} / V=L \sqrt{1-V^{2} / C^{2}} / V=t \sqrt{1-V^{2} / C^{2}}$, put it into Formula (15),

$$
Y=\frac{F t^{2}}{2 m \sqrt{1-V^{2} / C^{2}}}\left(1-V^{2} / C^{2}\right)=\frac{F t^{2}}{2 m} \sqrt{1-V^{2} / C^{2}}
$$

It is obvious that the relationship formula is the same as real deflection distance (2).

For the above analysis, the deflection results of charged particles moving at high speed got by $Z^{\prime}$ and $Z$ shall be the same, which is in compliance with Relativity Principle.

\section{Application Scope of Lorentz Force}

I hereby point out that the force on Q will be the same only in the uniform magnetic field through the analysis with the help of electric magnetic field and Lorentz Force; under normal condition, the two results derived from the two methods will not be the same in non-uniform magnetic field.

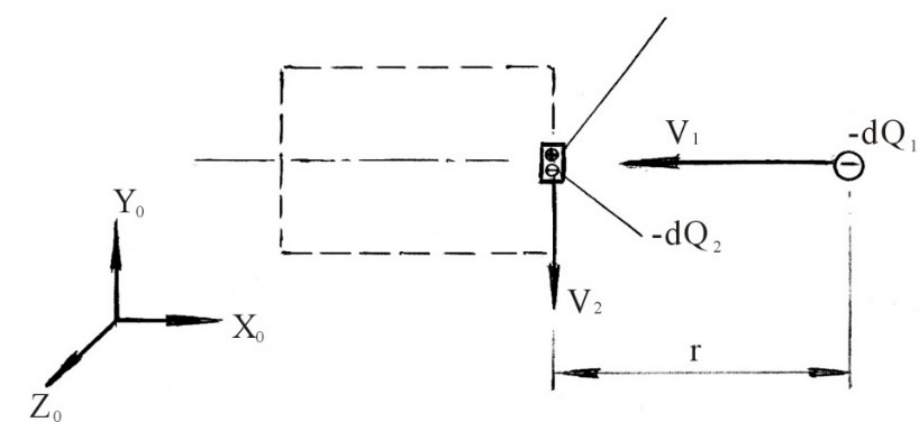

Figure 2. Analysis on interaction between moving electric charge and current component

Figure 2, electric charge $-d Q_{1}$ is moving towards $I d l$ at speed $V_{1}$, the capacity of negative electron moving in $I d l$ is $-d Q_{2}$ and velocity speed of $-d Q_{2}$ is $V_{2}$ (contrary direction with I), the capacity of positive electron in $I d l$ with the same capacity of that in $-d Q_{2}$ is $-d Q_{2}$ 。

First, the interaction force between $-d Q_{1}$ and $I d l$ is subject to analysis based on electric field. Since the relative velocity between negative charges or negative charge and positive charge in any relative motion is identical in magnitude and contrary in direction, The shrinkage condition observed each other is completely similar; therefore the force on each other is the same as that when they are relatively static. It is certain that they are the same in force and contrary in direction. We can know from this that both the interaction between $-d Q_{1}$ and $-d Q_{2}$ and that between $-d Q_{1}$ and positive electron $d Q_{2}$ are equal in force and contrary in direction; namely, the inter-force is naturally equal with contrary direction. It is obvious that the analysis result for electric field is in compliance with reaction law.

It is observed from analysis of interaction between $-d Q_{1}$ and $I d L$ based on Lorentz Force that only $-d Q_{2}$ in motion in current element generates magnetic field; since $d Q_{2}$ is in no motion, accordingly, it generates no magnetic field.

The electric field strength generated by $-d Q_{2}$ in $-d Q_{1}$ is $d \boldsymbol{E}_{2}$, the following $\boldsymbol{X}_{0}, \boldsymbol{Y}_{0}$ and $\boldsymbol{Z}_{0}$ are unit vectors, the motion velocity of $d \boldsymbol{E}_{2}$ is $\boldsymbol{V}_{2}, \boldsymbol{V}_{2}$ is referred to as $V_{2}\left(-\boldsymbol{Y}_{0}\right)$, according to the previous Formula (4), the magnetic induction strength generated by $d E_{2}\left(-\boldsymbol{X}_{0}\right)$ in $-d Q_{1}$ is as follows:

$$
\begin{aligned}
d \boldsymbol{B}_{2} & =\frac{1}{C^{2}} \boldsymbol{V}_{2} \times d \boldsymbol{E}_{2}=\frac{1}{C^{2}} V_{2}\left(-\boldsymbol{Y}_{0}\right) \times d E_{2}\left(-\boldsymbol{X}_{0}\right) \\
& =\frac{1}{C^{2}} V_{2} d E_{2}\left(-\boldsymbol{Z}_{0}\right)
\end{aligned}
$$

The motion velocity of $-d Q_{1}$ is $\boldsymbol{V}_{1}, \boldsymbol{V}_{1}$ is also referred to as $V_{1}\left(-\boldsymbol{X}_{0}\right)$, accordingly, Lorentz Force on $-d Q_{1}$ is as follows:

$$
d \boldsymbol{F}_{1}=-d Q_{1} \boldsymbol{V}_{1} \times d \boldsymbol{B}_{2}=\frac{-d Q_{1}}{C^{2}} V_{1}\left(-\boldsymbol{X}_{0}\right) \times V_{2} d E_{2}\left(-\boldsymbol{Z}_{0}\right)
$$




$$
=\frac{-d Q_{1}}{C^{2}} V_{1} V_{2} d E_{2}\left(-\boldsymbol{Y}_{0}\right)=\frac{1}{C^{2}} V_{1} V_{2} d E_{2} d Q\left(\boldsymbol{Y}_{0}\right)
$$

With regard to Lorentz Force on $-d Q_{2}$, the electric field strength generated by $-d Q_{1}$ in $-d Q_{2}$ is $d E_{1}\left(\boldsymbol{X}_{0}\right)$, the magnetic induction strength generated by $d E_{1}\left(\boldsymbol{X}_{0}\right)$ in $-d Q_{2}$ is as follows:

$$
d \boldsymbol{B}_{1}=\frac{1}{C^{2}} V_{1}\left(-\boldsymbol{X}_{0}\right) \times d E_{1}\left(\boldsymbol{X}_{0}\right)=0
$$

Since positive charge $d Q_{2}$ in no motion, therefore $d Q_{2}$ is not subject to Lorentz Force, the Lorentz Force on current element $I d L$ is the Lorentz Force on $-d Q_{2}$, the Lorentz Force on $-d Q_{2}$ is as follows:

$$
d \boldsymbol{F}_{2}=-d Q_{2} V_{2}\left(-\boldsymbol{Y}_{\boldsymbol{0}}\right) \times d \boldsymbol{B}_{1}=0 \quad\left(d \boldsymbol{B}_{1}=0\right)
$$

It is observed from comparison between action $d \boldsymbol{F}_{1}$ and reaction $d \boldsymbol{F}_{2}$ that they are different in magnitude and identical in direction; that is to say that adopting Lorentz Force to analyze interaction force between charge in motion and current element results in action and reaction are different in magnitude and not contrary in direction; namely, the result is contrary to law of reaction. It is observed from further analysis that in addition to $\boldsymbol{V}_{1}$ in parallel with $\boldsymbol{V}_{2}$ as motion velocity of $\boldsymbol{V}_{1}$, it is observed from analysis of Lorentz Force along any direction of $V_{1}$ that the interaction and reaction between $-d Q_{1}$ and $I d l$ are different in magnitude and not contrary in direction, namely the results are contrary to the law of reaction.

It is stated that there is no isolated current element in the universe and it is impractical to adopt current element for analysis. It is also known that scientific theory necessitates both tenable macro-analysis and micro-analysis. In fact, many theories are based on from micro-analysis to macro-analysis. If micro-analysis fails to be tenable, there shall be an example showing that macro-analysis fails to be tenable. The micro-current element given in Figure 2 extends along the dotted line shown in the figure, becoming the macro-current carrying coil. Analysis of the interaction force between current carrying coil and $-d Q_{1}$ shown in dotted line in Figure 2, the inevitable analysis conclusion is that the interaction and reaction between charge in motion $-d Q_{1}$ and current carrying coil are not contrary in direction and different in magnitude. Reference document 2 details the analysis result.

According to the above mentioned analysis, it is concluded that Lorentz Force is only applicable to uniform magnetic field and not applicable to non-uniform magnetic field. In order to analyze the force on charge in motion in non-uniform magnetic field, the only way is to adopt acting force in electric field for analysis.

\section{References}

Purcell, E. M. (1979). Berkeley Physics Course. Science and technology of China press.

Wu, S. B. (1987). Fundamental of Relativity Theory. Shaanxi Science \& Technology Press.

Yao, K. (2012). Question on some principles of electromagnetism. Applied Physics Research, 4(3), p115.

Yao, K. (2013). Explanation of Electromagnetics by Motion of Electric Field LAP LAMBERT. Academic Publishing.

Yao, K. (2013). Set up invariable axiom of force equilibrium and solve problems about transformation of force and gravitational mass. Applied Physics Research, 5(1), p72.

Yao, K., \& Wang, Z. (2012). Inferring the fact that static magnetic field exists along with electrostatic field and conducting experimental verification in accordance with the theory of relativity. Applied Physics Research, $4(1), \mathrm{p} 222$.

\section{Copyrights}

Copyright for this article is retained by the author(s), with first publication rights granted to the journal.

This is an open-access article distributed under the terms and conditions of the Creative Commons Attribution license (http://creativecommons.org/licenses/by/3.0/). 